DOI 10. 18307/2018. 0501

(c) 2018 by Journal of Lake Sciences

\title{
三峡水库水环境相关研究文献计量分析与未来展望
}

\author{
郭劲松 $^{1,2}$, 陈海燕 ${ }^{1}$, 李 哲 ${ }^{1,2}$, 肖 艳 $^{2}$, 方 芳 $^{1}$ \\ ( 1 : 重庆大学城市建设与环境工程学院,重庆 400045) \\ (2: 中国科学院重庆绿色智能技术研究院水库水环境重点实验室,重庆 400714)
}

\begin{abstract}
摘 要: 自三峡工程论证以来,其建设和运行可能引发的问题成为相关领域研究的热点. 2003 年 6 月,三峡水库开始一期 蓄水. 检索发现, 2002 年之前有关三峡的研究绝大部分集中于移民和工程建设风险等方面, 与水环境相关的论文数量不 足 5\%,相对较少. 因此,本文重点对 2002-2017 年期间,在 SCI-E/SSCI 上刊发的 520 篇研究型英文论文和在中国核心期 刊上刊发的 777 篇中文论文进行计量分析. 结果表明: 关于三峡水库水环境的研究论文数量整体呈逐年递增趋势, 中国在 三峡水库水环境研究中占据主导地位; ENVIRONMENTAL SCIENCE AND POLLUTION RESEARCH 和《环境科学》分别是刊 发英文论文和中文论文最多的期刊; 中国科学院、重庆大学和三峡大学是三峡水库水环境研究最活跃的机构; “消落带”、 “水华”、“浮游植物”、“营养盐” 和 “水温分层” 等方面是三峡水库水环境近几年研究的热点. 分析关键词还发现, 来自不 同学科背景的学者关注的兴趣点和重点不同. 一方面, 这有利于更全面的研究和多学科之间的碰撞交流; 另一方面, 即使 是表达同一事实, 由于学科差异而采用了不同的学术术语和关键词, 又妨碍了学科之间的顺畅交流. 因此, 从科学凝炼不 同类型水域共性问题和整合及运用多尺度、多维度的研究手段两个方面着手, 不仅可以深度融合不同学科的理论和学术 思想,还能深化三峡水环境的研究并提升我国在水库环境生态学研究领域的国际影响力.
\end{abstract}

关键词: 水环境; 富营养化;水华;营养盐;水动力;三峡水库

\section{Bibliometric and hot topic analysis of related literatures on water environment in Three Gorges Reservoir}

\section{GUO Jinsong $^{1,2}$, CHEN Haiyan ${ }^{1}$, LI Zhe ${ }^{1,2}$, XIAO Yan ${ }^{2} \&$ FANG Fang ${ }^{1}$}

(1: Faculty of Urban Construction and Environmental Engineering, Chongqing University, Chongqing 400045, P.R.China)

(2: Key Lab of Reservoir Aquatic Environment, Chongqing Institute of Green and Intelligent Technology, Chinese Academy of Sciences, Chongqing 400714, P.R. China)

Abstract: Since the impoundment of Three Gorges Reservoir, the problem caused by the construction and operation of Three Gorges Reservoir has become a hot topic in related fields. In June 2003, the Three Gorges Reservoir started the impoundment. Search found that, before 2002, most of the Three Gorges related research focused on the Three Gorges migrants, the Three Gorges project construction risk, while number of water environment related papers is relatively small, less than $5 \% .520$ articles published in SCI-E/SSCI and 777 articles published on Chinese core journals from 2002 to 2017 areanalyzed based on the bibliometrics method. The results showed that the number of research papers of Three Gorges Reservoir increased, and China plays a dominant role in the research of Three Gorges Reservoir. ENVIRONMENTAL SCIENCE AND POLLUTION RESEARCH and ENVIRONMENTAL SCIENCE publish the most amount of the papers in English and Chinese, respectively. Chinese Academy of Sciences, Chongqing University and China Three Gorges University are the most active institutions in the study of water environment about the Three Gorges Reservoir. "banded zone”, “algal bloom”, “phosphorus form”, “water temperature stratification” and so on are the focus of the Three Gorges Reservoir in the past several years. Analysis of keywords also found that scholars from different disciplines focus on different points of interest and focus. On the one hand, it is conducive to the exchange of collisions and more comprehensive studies among different disciplines. On the other hand, even if the same facts are expressed, different academic terms and keywords are used due to different disciplines, which hinders interdisciplinary communication. Therefore, starting with the common problem of

* 国家自然科学基金项目 (51679226,41771520) 资助. 2018-01-11 收稿; 2018-01-20 收修改稿. 郭劲松 ( 1963 ), 男,博士,教授;E-mail:guo0768@ cqu.edu.cn. 
scientifically practicing different types of waters and integrating and using multi-scale and multi-dimensional research methods, can not only deeply integrate the theory and academic thought of different disciplines, but also study the water environment of the Three Gorges and enhance our country's international influence in reservoir environmental ecology research.

Keywords: Water environment; entrophication; algal bloom; nutrient; hydrodynamic; Three Gorges Reservoir

三峡工程是目前世界上最大的水利水电枢纽工程, 也是综合治理长江中下游洪涝灾害的关键性控制工 程,兼具防洪、发电、航运、灌溉、供水和发展库区经济等巨大的综合经济效益. 2003 年 6 月, 三峡工程开始一 期蓄水;2006 年 9 月,三峡工程实行第二期蓄水;2010 年 10 月,三峡工程首次蓄水至 $175 \mathrm{~m}$ 设计水位. 三峡 工程蓄水后, 由于干流回水顶托, 造成支流库湾水质恶化, 藻类水华频繁暴发. 因此, 与三峡水库相关的水环 境、水生态以及水华问题引起国内外广泛关注. 对三峡水库水环境相关的研究文献进行全面统计计量分析, 可以较系统地总结回顾已有研究成果, 为更深人地开展三峡水库水环境研究提供参考.

\section{1 文献来源}

为全面掌握三峡水库水环境研究进展, 本研究不仅分析中文论文, 也分析英文论文. 检索 2002 年之前 三峡水库水环境相关研究论文, 可以发现, 不仅论文数量较少, 而且研究内容大部分是关于三峡水库移民、 三峡工程建设风险等的探讨, 所以, 本文主要分析 2002 年之后三峡水库水环境相关研究论文. 本研究中的 英文论文来源于 Web of Science ${ }^{\mathrm{TM}}$ 核心合集的 Science Citation Index Expand (SCI-Expand) 和 Social Sciencs Citation Index ( SSCI) 数据库, 主题检索式为 “TS = ( 'Three Gorge *' OR '3 Gorge *') AND ( 'water environment' OR 'algae' OR ' algal bloom *' OR 'entrophication *' OR 'water temperature' OR 'phytoplankton' OR 'nutritive salt' OR ' greenhouse gas *') , 检索年份为 2002-2017 年; 中文论文来源于中国学术期刊网络 数据总库 (中国知网, $\mathrm{CNKI}$ ), 主题检索式为“ $\mathrm{SU}=$ “三峡” and $\mathrm{SU}=($ “水环境” + “藻类” + ‘水华” + ‘水温” + “浮游植物” ‘营养盐’ ‘温室气体” )”, 检索年份也是 2002-2017 年, 检索范围限制为核心期刊. 根据上 述检索式共检索到英文论文 534 篇和中文论文 777 篇, 其中, 英文论文包含 520 篇研究文章 (Artical)、1 篇快 报 (Letter) 和 13 篇综述 (Review). 考虑到文献类型在分析中的重要性, 选择研究型论文进行分析, 所以最终 选取 520 篇研究型英文论文和 777 篇中文论文进行分析. 为了方便进一步分析, 将上述 1297 篇中、英文论文 的相关数据和信息导人 Excel 构建完成文献信息数据库.

\section{2 论文发表的年度与期刊分布}

\section{1 论文发表的年度变化}

2002-2017 年期间, 三峡水库水环境研究的中、英文论文数量总体上呈现上升趋势 (图 1), 表明三峡水 库在过去十几年逐渐受到国内外广泛关注. 英文论文方面, 2002-2005 年公开发表在 SCI-E/SSCI 期刊上的 论文比较少, 在不同期刊上发表的总共 17 篇. 其中. Liu 等 ${ }^{[1]} 2003$ 年在 BIOGEOCHEMISTRY 上发表的“Nutrients in the Changjiang and its tributaries”, 讨论了长江及其支流中的溶解态和颗粒态, 以及有机态和无机态的 氮、磷、硅, 分析了三峡工程建成后对长江口以及其邻近海域的可能影响. 该文被引频次高达 144 , 是本研究 涉及的所有文献中被引次数最多的英文论文. 综合分析, 三峡水库在 2002-2005 年未引起广泛关注. 2006 年 9 月,三峡工程实行二期蓄水至 $156 \mathrm{~m}$ 水位, 标志着三峡工程进人初期运行阶段. 随着三峡工程的运行, 三峡水库引起学者的关注,2006 年之后, 三峡水库水环境研究的英文论文呈递增趋势, 特别是近几年, 相关 文献激增, 2016 年发文量高达 83 篇,2017 年达到 79 篇.

中文论文方面, 2002-2017 年期间, 论文数量呈现先增加后减少的趋势, 在 2012 年达到峰值 83 篇. 2002-2012 年递增的趋势主要是因为随着三峡水库的运行, 库区所暴露出来的问题推动了三峡水库水环境 的相关研究; 笔者认为 2012-2017 年减少的趋势是因为: 三峡水库不仅是国内的研究热点, 也成为了国际上 的研究热点,所以促使更多的研究成果发表在外文期刊,以方便各国学者的交流讨论.

\section{2 主要发文期刊}

分析的 520 篇研究型英文论文发表在 233 个国际期刊上,其中,有 140 个期刊只发表过 1 篇论文,有 83 


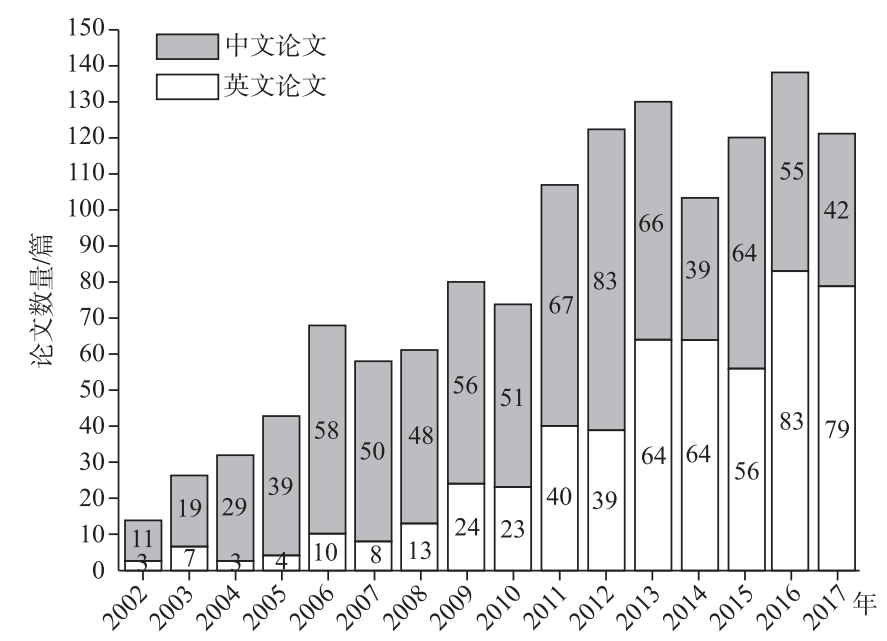

图 $12002-2017$ 年关于三峡水库水环境研究的 SCI-E/SSCI 和中文核心期刊论文数量

Fig.1 Number of articles about the Three Gorges Reservoir published in SCI-E/SSCI and Chinese core journals (2002-2017)

个期刊的发文量为 2 7 篇, 10 个期刊的发文量 $\geqslant 8$ 篇. ENVIRONMENTAL SCIENCE AND POLLUTION RESEARCH 发表了 21 篇论文, ENVIRONMENTAL EARTH SCIENCES 和 SCIENCE OF THE TOTAL ENVIRONMENT 均发表 17 篇论文, 这 3 个期刊共发表 55 篇论文, 占英文总发文量的 $10.58 \%$. 在 10 个发文 量较多的国际期刊中,荷兰和德国均有 3 个,中国有 2 个,英国和美国各有 1 个.

相对于英文论文而言, 本研究分析的 777 篇中文论文发表的期刊相对集中,共发表在 145 个中文期刊 上. 其中, 22 个期刊的发文量 $\geqslant 10$ 篇, 61 个期刊的发文量 $<10$ 篇, 62 个期刊只发表了 1 篇论文. 总发文量排 前 10 名的期刊共发文 361 篇, 占总发文量的 $46.46 \%$. 《环境科学》发文量最多, 高达 67 篇; 《人民长江》和 《长江流域资源与环境》的发文量分别为 62 和 52 篇.

\section{3 研究力量分析 : 相应国家地区机构及相应作者}

\section{1 英文论文主要国家及地区分布}

分析英文论文的发文国家可得,520 篇研究型英文论文由 35 个国家和地区完成,其中,由一个国家或地 区独立完成的论文共 356 篇,占论文总量的 68.46\%.中国大陆独立完成论文 310 篇, 美国独立完成 13 篇, 澳 大利亚、中国台湾地区各独立完成 5 篇, 中国大陆独立完成的论文量遥遥领先. 因为三峡水库对于长江流域 的国计民生和生态安全有重要影响, 所以大陆学者对于三峡水库水环境研究更加关注. 大部分国家或地区 是通过与其他国家或地区合作的形式参与三峡水库的研究, 而与中国大陆的合作最多, 高达 136 篇. 由图 2 可以看出, 中国处于各国家或地区合作的中心位置, 与美国、德国和加拿大等国都有紧密的合作关系. 因为 国内学者更容易获得三峡水库的相关资料与数据, 所以其他国家或地区大部分的研究是与中国学者合作来 完成的.

\section{2 主要研究机构}

表 1 列出了三峡水库水环境研究论文的主要研究机构统计结果, 从各机构总的发文量和作为第一署名 机构的发文量两方面给出了各机构的英文论文贡献和中、英文论文的总贡献. 由表 1 可知,三峡水库水环境 研究的主要机构绝大部分来自中国. 中国科学院无论是论文总数, 还是作为第一机构发表的论文数均是研 究论文贡献最多的机构. 重庆大学、三峡大学和西南大学不仅关于三峡水库水环境研究的总发文量名列前 茅, 而且作为第一机构的发文量排名也很靠前. 综合来看, 各机构的总发文量和作为第一研究机构的发文量 排名没有太大的变化, 但英文论文占比却有比较大的差异. 一个机构的英文论文占比与它的国际化程度、英 


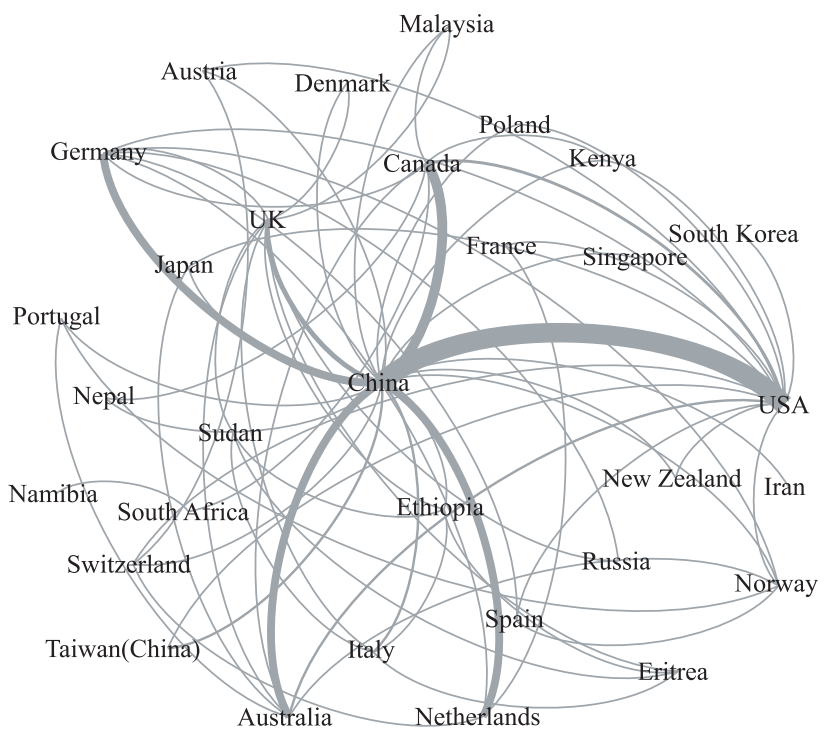

图 2 三峡水库水环境研究的国家地区合作网络图

(两个国家之间有连线, 表示两国之间有合作, 连线越粗, 则表示合作越紧密, 连线的长短没有特殊含义)

Fig. 2 The cooperation network of the countries/territories doing the research about the water environment of the Three Gorges Reservoir

文水平等有很大关系, 中国科学院、河海大学和武汉大学的英文论文占比总体比较靠前, 表明这 3 个机构国 际化程度较高. 国外的机构对于三峡水库水环境研究也有较大贡献, 比如加拿大的里贾纳大学、德国卡尔斯 鲁厄理工学院以及美国的加利福尼亚大学,他们在三峡水库水环境研究方面分别发表了 $6 、 4$ 和 3 篇论文.

表 1 三峡水库水环境研究论文的主要研究机构 *

Tab.1 Major research agencies contributing to the articles about the Three Gorges Reservoir

\begin{tabular}{ccc}
\hline 机构 & 总论文数量/篇 & 第一研究机构论文数量/篇 \\
\hline 中国科学院 & $323(181)$ & $235(129)$ \\
重庆大学 & $171(40)$ & $141(27)$ \\
三峡大学 & $112(37)$ & $85(20)$ \\
西南大学 & $99(20)$ & $77(15)$ \\
武汉大学 & $67(23)$ & $31(15)$ \\
中国环境科学研究院 & $61(16)$ & $30(3)$ \\
河海大学 & $54(28)$ & $36(21)$ \\
重庆市环境科学研究院 & $40(3)$ & $26(1)$ \\
湖北工业大学 & $39(8)$ & $5(0)$ \\
中国长江三峡集团公司 & $32(5)$ & $4(0)$
\end{tabular}

* 括号外是中、英文论文总数,括号内为英文论文数.

\section{3 主要研究作者}

从事三峡水库水环境研究的研究者较多, 仅中文论文涉及的研究者就高达 1650 位, 因英文文献中本为 不同作者但英文名字 (拼音) 拼写相同的情况较多, 所以本研究未分析英文论文涉及的总研究者. 以第一作 者身份发表中、英文总量排前 5 名的依次是中国科学院 (重庆大学) 的李哲、北京大学的王丽婧、武汉大学的 杨正健、重庆大学 (中国科学院) 的郭劲松以及三峡大学的王海云. 以第一作者身份发表英文论文总量排前 6 名的依次是北京大学的王丽婧、中国科学院 (重庆大学) 的李哲、武汉大学的杨正健、三峡库区生态环境教 
育部工程研究中心的宋林旭、三峡大学的王海云和重庆大学的张智 (其中王海云和张智以第一作者身份发 表英文论文均为 1 篇, 并列第 5). 中、英文论文发文总量排前 5 名的依次是三峡大学的刘德富、重庆大学 (中 国科学院) 的郭劲松、中国科学院的蔡庆华、中国科学院 (重庆大学) 的李哲以及武汉大学的杨正健. 英文论 文发文总量排名前 5 的依次是中国科学院的蔡庆华、中国科学院的毕永红、三峡大学的刘德富、中国科学院 的胡征宇以及中国环境科学研究院的郑丙辉.

对三峡水库水环境研究主要作者的分析表明, 三峡水库水环境研究以团队合作为主要方式, 贡献比较 大的作者团队主要有 4 个, 分别是武汉大学和三峡大学合作的杨正健和刘德富团队、重庆大学与中国科学 院合作的郭劲松团队、中国科学院的蔡庆华团队和中国科学院的胡征宇团队. 杨正健和刘德富团队侧重于 研究三峡水库香溪河, 主要研究香溪河水动力特征及其对浮游植物及水华的影响 ${ }^{[2-10]}$; 郭劲松团队重点关注 小江 (澎溪河) 的水环境及藻类多样性, 主要研究水环境中藻类和营养物的生物地球化学过程与机理, 也涉 及水库温室气体和碳循环 ${ }^{[1-19]}$; 中国科学院的蔡庆华团队的研究主要关注三峡水库香溪河库湾的微环境及 水生生物, 也通过建立模型预测监控水华 ${ }^{[20-26]}$; 中国科学院的胡征宇和毕永红团队则重点研究三峡水库调 度运行对藻类生态与水华暴发的影响, 以及三峡水库库区的有机污染物 ${ }^{[27-33]}$.

表 2 给出了三峡水库水环境相关研究主要作者已发表论文的被引频次信息. 中文论文篇均被引频次最 高为 46.71 , 最低为 9.47 ; 英文论文篇均被引频次最高为 22.00 , 最低为 1.00 . 其中, 中国科学院的蔡庆华发表 中、英文论文总量为 51 篇, 排名第 8 , 但中文论文篇均被引频次高达 46.71 , 排名第 1 , 英文论文篇均被引频次 为 12.44 , 排名第 5 . 就中、英文论文篇均被引频次而言, 中国科学院的蔡庆华团队位居榜首, 其次是三峡大学 和武汉大学合作的杨正健、刘德富团队以及中国科学院的胡征宇团队. 总体而言, 三峡水库水环境相关研究 中, 中文论文被引频次整体高于英文论文被引频次, 但均不太高, 这可能是因为关注三峡水库水环境的学者 拥有不同的学科背景, 他们关注的兴趣点和重点不一样, 所以彼此之间的对比较少; 其次, 这也可能是因为 目前三峡水库水环境研究学术成果仍然在一个积累过程中.

表 2 三峡水库水环境相关研究主要作者的论文被引频次分析

Tab.2 Citation frequency analysis of the main authors of the water environment related study of the Three Gorges Reservoir

\begin{tabular}{cccccccc}
\hline 作者 & 单位 & $\begin{array}{c}\text { 中文发 } \\
\text { 文量/篇 }\end{array}$ & $\begin{array}{c}\text { 总被引 } \\
\text { 频次 }\end{array}$ & $\begin{array}{c}\text { 篇均被 } \\
\text { 引频次 }\end{array}$ & $\begin{array}{c}\text { 英文发 } \\
\text { 文量/篇 }\end{array}$ & $\begin{array}{c}\text { 总被引 } \\
\text { 频次 }\end{array}$ & $\begin{array}{c}\text { 篇均被 } \\
\text { 引频次 }\end{array}$ \\
\hline 刘德富 & 三峡大学 & 59 & 848 & 14.37 & 16 & 209 & 13.06 \\
郭劲松 & 重庆大学/中国科学院 & 43 & 709 & 16.49 & 11 & 83 & 7.55 \\
蔡庆华 & 中国科学院 & 24 & 1121 & 46.71 & 27 & 336 & 12.44 \\
李哲 & 重庆大学/中国科学院 & 38 & 640 & 16.84 & 8 & 31 & 3.88 \\
杨正健 & 武汉大学 & 32 & 555 & 17.34 & 9 & 101 & 11.22 \\
纪道斌 & 三峡大学 & 32 & 515 & 16.09 & 9 & 86 & 9.56 \\
郑丙辉 & 中国环境科学研究院 & 27 & 1023 & 37.89 & 14 & 64 & 4.57 \\
胡征宇 & 中国科学院 & 21 & 887 & 42.24 & 15 & 99 & 6.60 \\
毕永红 & 中国科学院 & 13 & 212 & 16.31 & 17 & 85 & 5.00 \\
方芳 & 重庆大学 & 22 & 669 & 30.41 & 4 & 24 & 6.00 \\
王丽婧 & 北京大学 & 15 & 142 & 9.47 & 7 & 29 & 4.14 \\
张星 & 重庆市环境科学研究院 & 17 & 655 & 38.53 & 1 & 1 & 1.00 \\
陈永柏 & 中国长江三峡工程开发总公司 & 17 & 187 & 11.00 & - & - & - \\
李崇明 & 重庆市环境科学研究院 & 13 & 547 & 42.08 & 1 & 22 & 22.00 \\
邓春光 & 重庆市环境科学研究院 & 12 & 440 & 36.67 & - & - & - \\
\hline
\end{tabular}

“一”表示该项数据没有. 


\section{4 主要研究热点分析}

\section{1 中、英文论文关键词分析}

为了更好地把握三峡水库水环境研究的热点和趋势, 本研究对统计所关注的中、英文论文关键词进行 进一步分析. 选取 2002-2017 年在 SCI-E/SSCI 上发表的 520 篇英文论文和在中文核心期刊上发表的 777 篇中文论文,合计 1297 篇论文. 其中, 有 96 篇英文论文没有设置关键词, 对设置了关键词的 1201 篇论文的 关键词进行深人分析, 共获得 1850 个中文关键词和 1659 个英文关键词. 为了更好地整合分析关键词, 排除 三峡水库、三峡库区、三峡和三峡大坝等关键词.

对关键词信息进行分析,不难发现, 中文关键词和英文关键词排前 10 名的有 5 个是相同的,分别是香溪 河库湾、富营养化、长江、水华、水质, 这表明这些方面是国内外学者都比较关注的. 此外, 分析关键词信息还 可以得出来自不同学科的研究者在关注三峡水库水环境相关研究,他们从不同角度,用不同方法研究三峡 水库水环境的不同方面. 对高频的中英关键词进行分类分析, 可以更好地了解三峡库区的水环境研究,表 3 列出了主要中英关键词的 4 大分类, 分别是主要研究区域、水环境质量指标、主要研究藻类和主要研究方法. 主要研究区域方面, 中、英文关键词重合的比例很大, 均比较关注香溪河库湾、鄱阳湖、洞庭湖; 水环境质量 指标方面, 中、英文论文均比较关注氮磷营养盐、悬浮物和水温分层等; 主要研究藻类方面, 中文论文关注的 藻类相对多一些, 如蓝藻、拟多甲藻、冠盘藻、硅藻、附石藻类、绿藻、铜绿微囊藻、蛋白核小球藻等, 英文论文 则主要关注微囊藻和蓝藻; 在主要采用的研究方法方面: 中文论文主要采用数值模拟、二维水质模型、BP 神 经网络、三维水流模型等方法,英文论文主要采用数值模拟、遗传算法、小波分析、水质模型等.

表 3 三峡水库水环境研究的 SCI-E/SSCI 和中文核心期刊论文的主要关键词分类

Tab.3 Classification of the main keywords of the articles about the Three Gorges Reservoir published in SCI-E/SSCI and Chinese core journals

\begin{tabular}{|c|c|c|}
\hline 主要关键词分类 & 代表关键词 & 英文关键词 \\
\hline 主要研究区域 & $\begin{array}{l}\text { 香溪河库湾、小江回水区、洞庭湖、鄱阳湖、 } \\
\text { 大宁河、澎溪河、汉丰湖、乌江、长江 }\end{array}$ & $\begin{array}{l}\text { Yangtze River、Xiangxi Bay、Dongting Lake、Poyang } \\
\text { Lake、Pengxihe }\end{array}$ \\
\hline 水环境质量指标 & $\begin{array}{l}\text { 叶绿素 a、营养盐、重金属、水温分层、氮、 } \\
\text { 磷、录、悬浮物、COD }\end{array}$ & $\begin{array}{l}\text { Phosphorus 、nitrogen 、 suspended solids, heavy metals } \\
\text { water temperature stratification }\end{array}$ \\
\hline 主要研究藻类 & $\begin{array}{l}\text { 蓝藻、拟多甲藻、冠盘藻、硅藻、附石藻类、 } \\
\text { 甲藻、绿藻、铜绿微囊藻、蛋白核小球藻 }\end{array}$ & Microcystis、cyanobacteria \\
\hline 主要研究方法 & $\begin{array}{l}\text { 数值模拟、二维水质模型、BP 神经网络、三 } \\
\text { 维水流模型、WASP 生态动力学模型、一维 } \\
\text { 水动力模型 }\end{array}$ & $\begin{array}{l}\text { numerical simulation 、 genetic algorithm、 progressive } \\
\text { optimality algorithm 、 uncertainty analysis } \text { wavelet a- } \\
\text { nalysis water quality model }\end{array}$ \\
\hline
\end{tabular}

\section{2 近几年研究热点分析}

论文的关键词不仅高度概括了一篇论文的研究内容, 更反映了相关领域的研究热点. 为了分析三峡水 库水环境研究近几年的研究热点和趋势,本研究对 2012-2017 年的中、英文论文关键词进行分析.

分析中文关键词可以发现, “消落带”、“水华”和“浮游植物”在近 5 年出现的频率远高于 5 年前, 而“遥 感技术”、“沉积物”等是近 5 年才出现的, 说明研究者近几年比较关注这些研究领域. 三峡水库的运行在库 岸形成了垂直落差 $30 \mathrm{~m}$ 、总面积 $440 \mathrm{~km}^{2}$ 的消落带, 对消落带的研究探讨成为了近几年的热点. 研究涉及三 峡干支流主要的消落带, 分析研究了消落带土壤、沉积物的理化性质, 总氮、总磷以及氮磷各种形态的含量、 时空分布特征, 各形态氮磷之间及各形态动态、与土壤理化特征之间的相关性以及水动力条件和人类活动 对消落带土壤氮磷形态动态的影响等 ${ }^{[34-41]}$. 三峡水库蓄水之后, 由于水库回水顶托, 造成了支流库湾水质恶 化,水华频发. 水华和藻类群落结构及其防控是另一个研究热点, 研究者从水华现象与过程、水华期间藻类 群落结构、营养盐状况与水华的相关性等角度进行探讨 ${ }^{[9,27,42-45]}$. 上述研究中, 水温分层、模型、遥感技术等 也是高频率出现的关键词, 研究者既把它们作为研究的内容, 同时又作为研究三峡水环境问题的方法和手 段,甚至是运行和管理的手段在探讨应用 ${ }^{[43-49]}$. 
近五年来,关于三峡水环境研究英文论文高达 346 篇,占本文关注的英文论文总量的 $66.54 \%$,这说明近 几年三峡水库水环境的研究开始走向国际化. 分析英文关键词可以发现, 大部分关键词是与中文论文关键 词相重合的, “eutrophication”、“algal bloom”、“phytoplankton”、“nitrogen”、“remote sensing”、“phosphorus”是 高频出现的关键词, 有的是近 5 年才出现的. 这说明两个方面的问题:其一, 中、英文论文的主要作者群体是 一致的; 其二, 关于水库水环境问题国际国内关注点有重要的重合度, 能够得到国际学界的认同, 其影响力 正在扩大.

\section{3 未来研究展望}

分析中、英文论文的关键词还发现, 三峡水库水环境相关研究涉及的面越来越广,这说明关注三峡的学 者越来越多. 另一方面也因为关注三峡水环境的学者来自不同的学科, 拥有不同的学科背景, 因此关注的兴 趣点和重点不同. 不同学科之间的这种交流碰撞, 对于三峡水环境的研究是有利的. 与此同时我们也发现另 外一个现象, 就是即使是表达同一个事物, 由于不同学科的差异而采用了不同的学术术语和关键词, 因此, 这又妨碍了不同学科之间的顺畅交流, 也不利于中国学者关于水库水环境研究的整体实力在国际上的展示. 所以深度地有机融合不同学科的理论和学术思想, 发展具有中国特色的水库环境生态学, 形成一个新的学 科体系, 对提升我国在水库环境生态学研究领域的国际影响力, 既是挑战也是机遇. 笔者认为这样的机遇可 能包括以下几个方面:

1) 重视三峡研究的国际化,有利于我国在这方面引领地位的确立:由前文分析可知,我国在三峡研究的 国际合作方面处于中心地位, 在英文论文发文量和增长方面近 5 年开始快速走向国际. 如何把握机遇把这 种数量优势转化为水库水环境或水库湖沼学研究的学术地位优势值得思考. 笔者初浅的认为, 在气候变化 的大背景下, 清洁能源和可再生能源是减缓温室效应的重要选项和路径. 利用水能资源 (自然可再生能源) 筑坝蓄水发电既提供清洁能源, 又可优化淡水资源分配并提供大运能的航运, 其综合低碳效应在未来相当 长的时期仍是不可替代的. 但水库修建对河流原有的生源物质生物地球化学过程和流域生态系统的负面影 响仍然不可忽略. 三峡水库作为中国第一大水库, 不仅具有其独特性, 也应当具有水库湖沼过程的共性规 律, 对全球水库水环境研究的启示不言而喻. 结合当前国际研究的趋势, 以下 4 方面应深化关注: (1)水库修 建和运行对原河流生源物质的表生过程和流域生态的影响; (2)流域梯级开发和人类社会经济活动胁迫下, 生态环境变化在时间和空间的累积效应; (3)水库库区水污染物质的多介质 (包括消落带) 环境过程、归趋及 其生态适宜性调控原理; (4)多目标需求 (trade-off)下, 水库水环境生态系统服务 ( ecosystem service) 功能提升 与适应性管理 (adaptive management). 上述方面不仅需要对当前研究进一步深化, 也需要在横向上进行多学 科交叉融合和拓展.

2)对不同类型水域共性问题的科学凝炼:近年来, 不同类型水域生态环境的独特性越来越受到湖沼学家 的重视, 相继发展了浅水湖泊、河流、湿地、地下水、水库等水文单元的生态环境研究体系, 并力图通过分析生 态环境的服务功能, 指导人类开发活动与环境管理. 尽管如此, 人为划分的水文生态区系单元并无法阻碍它们 之间必然的自然联系, 学科研究对象的分化并不意味着它们之间的鸿沟将逐渐扩大, 而事实上研究对象与内 容的分化、细化恰恰是为了未来更系统的整合. 由于陆表水文循环和过程依然是水库一河流系统水生态环境变 化的动力基础,尽管水库构建了不同类型的水环境区系, 但是水库、湖泊、河流、地下水等各水文单元之间存在 着的能量、物质和信息交换并没有终止,相反它们之间在边界上的相互作用更强、影响更密切,因此,寻找不同 水文水环境体系的共性规律将成为未来水库生态环境学发展的重要方向. 近十年来国际上, 生态水文学 ( ecohydrology)、生态化学计量学 (ecological stoichiometry) 日渐兴起也许可为此突破提供重要参考.

3) 多尺度、多维度研究手段的整合与运用: 水环境与生态学研究发展至今,形成了从基因水平到全球水 圈水平的庞大学科体系, 研究对象的空间尺度从埃 $(\AA)$ 跨越到光年, 时间尺度从飞秒、皮秒延伸至亿年以上, 其研究所涉及时空尺度已涵盖了目前生命活动所能够涵盖到的所有范围. 但是, 科学研究是以特定对象为 基础而开展的. 针对不同组织层级开展的研究并发展相适配的研究方法学是目前普遍的研究思路,但不同 组织层级间所关注的尺度和研究方法手段的差异很可能造成各层级规律认识存在较大差异和误读, 诸如区 群翏误 (ecological fallacy) 等生态研究方法学上的问题难以避免. 因此, 水环境与生态学研究的方法、手段如 何从宏观深人至微观、如何从微观走向宏观, 以实现系统的整合, 这可能成为今后相当长一段时间内环境与 
生态学研究发展中不可忽略的科学方法论, 也将对水库水环境研究的深化与发展起到引领性的影响.

\section{5 结论}

综上所述, 可以得到以下结论: 1) 2002-2017 年期间, 三峡水库水环境研究论文数量总体呈上升趋势, 中文核心期刊论文数量呈先增加后减少的趋势, SCI-E/SSCI 期刊论文数量则总体呈现上升趋势; 2) 刊发三 峡水库水环境研究论文杂志众多, 其中, ENVIRONMENTAL SCIENCE AND POLLUTION RESEARCH 和《环境 科学》分别是刊发英文论文和中文论文最多的期刊;3) 中国科学院、重庆大学、三峡大学和西南大学是三峡 水库水环境研究领域较活跃的机构, 三峡水库相关的研究以团队合作为主;4) 三峡水库水环境研究的热点 有“消落带”、“水华”、“浮游植物”、“营养盐”和“水温分层”、“沉积物”等方面.

三峡水库水环境研究呈现出多学科、多视角齐头并进的活跃局面, 但多学科融合的深度和整体系统性 还不够. 三峡水库属于典型的峡谷河道型水库,其生态系统在物理环境上介于河流和天然湖泊之间, 其生态 格局则呈现出上述两种状态间的过渡性特征, 并在水库运行的干扰下具有独特性. 同时,在水库运行下其水 环境生态系统的发育、演变及其累积影响等方面尚需要长期系统跟踪研究. 现实方面, 三峡水库的建成对运 行维护和管理模式提出了更新、更高的要求. 因此,三峡水库为我国河流-水库环境学研究创造了新的契机, 搭建清晰的学科理论框架, 构建明确、完善的研究方法体系, 必将是未来水库环境生态学研究的努力方向.

\section{6 参考文献}

[ 1 ] Liu SM, Zhang J, Chen H et al. Nutrients in the Changjiang and Its Tributaries. Biogeochemistry, 2003, 62(1) : 1-18.

[ 2 ] Fang LJ, Liu DF, Yang ZJ et al. Succession of phytoplankton in Xiangxi Bay of Three-Gorge Reservoir in summer and its causes. Journal of Ecology and Rural Environment, 2013, 29(2): 234-240. [方丽娟, 刘德富, 杨正健等. 三峡水库香 溪河库湾夏季浮游植物演替规律及其原因. 生态与农村环境学报, 2013, 29(2) : 234-240.]

[ 3 ] Xiao SB, Liu DF, Wang YC et al. Temporal variation of methane flux from Xiangxi Bay of the Three Gorges Reservoir. Scientific Reports, 2013, 3(8): 1-8.

[ 4 ] Yang L, Liu DF, Huang YL et al. Isotope analysis of the nutrient supply in Xiangxi Bay of the Three Gorges Reservoir. Ecological Engineering, 2015, 77: 65-73.

[ 5 ] Huang YL, Fan DX, Liu DF et al. Nutrient estimation by HJ-1 satellite imagery of Xiangxi Bay, Three Gorges Reservoir, China. Environmental Earth Sciences, 2016, 75(8) : 1-13. DOI:10.1007/s12665-016-5421-z.

[ 6 ] Liu L, Liu DF, Johnson DM et al. Effects of vertical mixing on phytoplankton blooms in Xiangxi Bay of Three Gorges Reservoir: implications for management. Water Research, 2012, 46(7) : 2121-2130. DOI:10.1016/j.watres.2012.01.029.

[ 7 ] Yang ZJ, Liu DF, Ma J et al. Effects of special vertical layered water temperatures on algal bloom in Xiangxi Bay of Three Gorges Reservoir. Engineering Journal of Wuhan University, 2012, 45(1): 1-9, 15. [杨正健, 刘德富, 马骏等. 三峡水 库香溪河库湾特殊水温分层对水华的影响. 武汉大学学报: 工学版, 2012, 45(1): 1-9, 15.]

[ 8 ] Ji DB, Liu DF, Yang ZJ et al. The influence of the flood impoundment in Xiangxi Bay density current intrusion on water bloom. Journal of Hydraulic Engineering, 2010, 41(6): 691-696, 702. [ 纪道斌, 刘德富, 杨正健等. 汛末蓄水期香 溪河库湾倒灌异重流现象及其对水华的影响. 水利学报, 2010, 41(6) : 691-696, 702.]

[ 9 ] Xiao M, Li WM, Liu DF et al. Prediction of algal bloom variation in backwater areas of tributaries in Three-Gorges Reservoir based on multiple optimized grey model. Acta Scientiae Circumstantiae, 2017, 37(3): 1153-1161. [肖鸣, 李卫明, 刘德富等. 基于多重优化灰色模型的三峡库区香溪河支流回水区水华变化趋势预测研究. 环境科学学报, 2017, 37(3) : 1153-1161.]

[10] Ji DB, Wells SA, Yang Z et al. Impacts of water level rise on algal bloom prevention in the tributary of Three Gorges Reservoir, China. Ecological Engineering, 2017, 98: 70-81.

[11] Feng J, Li Z, Yan B et al. In situ growth rate of dominant algae species in Pengxi River of the Three Gorges Reservoir. J Lake Sci, 2014, 26(2) : 235-242. DOI:10.18307/2014.0210. [冯婧, 李哲, 间彬等. 三峡水库不同运行阶段澎溪河 典型优势藻原位生长速率. 湖泊科学, 2014, 26(2): 235-242.]

[12] Fang F, Zhou H, Li Z et al. Optical properties of water body in Xiaojiang River backwater area, Three Gorges Reservoir. Journal of Chongqing University: Natural Science Edition, 2011, 34 (9) : 102-108. [方芳, 周红, 李哲等. 三峡小江回 水区水体光学特征及分析. 重庆大学学报: 自然科学版, 2011, 34 (9) : 102-108.]

[13] Li Z, Guo JS, Fang F et al. Seasonal variation of Cyanobateria and its potential relationship with key environmental factors 
in Xiaojiang backwater area, Three Gorges Reservoir. Environmental Science, 2010, 31(2): 301-309. [李哲, 郭劲松, 方芳等. 三峡小江回水区蓝藻季节变化及其与主要环境因素的相互关系. 环境科学, 2010, 31(2) : 301-309.]

[14] Li Z, Wang S, Guo JS et al. Phytoplankton diversity in backwater area of the Pengxi River in the Three Gorges Reservoir before and after 156m impoundment. J Lakes Sci, 2012, 24(2) : 227-231. DOI: 10.18307/2012.0209. [李哲, 王胜, 郭 劲松等. 三峡水库 $156 \mathrm{~m}$ 蓄水前后澎溪河回水区藻类多样性变化特征. 湖泊科学, 2012, 24(2) : 227-231.]

[15] Li Z, Guo JS, Long M et al. Seasonal variation of nitrogen and phosphorus in Xiaojiang River-A tributary of the Three Gorges Reservoir. Frontiers of Environmental Science \& Engineering in China, 2009, 3(3) : 334-340.

[16] Li Z, Zhang ZY, Xiao Y et al. Spatio-temporal variations of carbon dioxide and its gross emission regulated by artificial operation in a typical hydropower reservoir in China. Environmental Monitoring and Assessment, 2014, 186(5) : 3023-3039. DOI: $10.1007 / \mathrm{s} 10661-013-3598-0$.

[17] Li Z, Zhang ZY, Lin CX et al. Soil-air greenhouse gas fluxes influenced by farming practices in reservoir drawdown area: A case at the Three Gorges Reservoir in China. Journal of Environmental Management, 2016, 181: 64-73.

[18] Yang $\mathrm{H}$, Yasarer $\mathrm{LMW}$, Zhe $\mathrm{L}$ et al. Air-water $\mathrm{CO}_{2}$, and $\mathrm{CH}_{4}$, fluxes along a river-reservoir continuum: Case study in the Pengxi River, a tributary of the Yangtze River in the Three Gorges Reservoir, China. Environmental Monitoring \& Assessment, 2017, 189(5): 223.

[19] Li Z, Lu LH, Lv PY et al. Carbon footprints of pre-impoundment clearance on reservoir flooded area in China's large hydro-projects: Implications for GHG emissions reduction in the hydropower industry. Journal of Cleaner Production, 2017, 168: 1413-1424.

[20] Ye L, Xu YY, Cai QH. The distribution of nitrate and phosphate in the Xiangxi Bay in spring. Acta Hydrobiologica Sinica, 2006, 30 (1) : 75-79. [ 叶麟, 徐耀阳, 蔡庆华. 香溪河库湾春季水华期间硝酸盐、磷酸盐的时空分布. 水生生物学 报, 2006, 30(1): 75-79.]

[21] Han XQ, Ye L, Xu YY et al. Dynamic analysis of a concentration and its influencing factors in Xiangxi Bay of chlorophyll a. Acta Hydrobiologica Sinica, 2006, 30(1) : 89-94. [ 韩新芹, 叶麟, 徐耀阳等. 香溪河库湾春季叶绿素 a 浓度动态 及其影响因子分析. 水生生物学报, 2006, 30(1): 89-94.]

[22] Xu YY, Cai QH, Li DF et al. Preliminary report on diel vertical distribution patterns of peridiniopsis in Xiangxi Bay of the Three Gorges Reservoir. Journal of Wuhan Botanical Research, 2008, 26(6) : 608-612. [徐耀阳, 蔡庆华, 黎道丰等. 三峡水库香溪河库湾拟多甲藻昼夜垂直分布初步研究. 武汉植物学研究, 2008, 26(6) : 608-612.]

[23] Shen HL, Cai QH, Shao ML et al. Community structure characteristics of phytoplankton along a cascade of reservoirs in Xiangxi River Basin, Three Gorges Reservoir. J Lake Sci, 2012, 24(2) : 197-205. DOI: 10.18307/2012.0205. [申恒伦, 蔡庆华, 郡美玲等. 三峡水库香溪河流域梯级水库浮游植物群落结构特征. 湖泊科学, 2012, 24(2) : 197-205.]

[24] Ye L, Cai QH, Zhang M et al. Real-time observation, early warning and forecasting phytoplankton blooms by integrating in situ automated online sondes and hybrid evolutionary algorithms. Ecological Informatics, 2014, 22: 44-51.

[25] Xu YY, Wang L, Cai QH et al. Temporal coherence of chlorophyll a during a spring phytoplankton bloom in Xiangxi Bay of Three-Gorges Reservoir, China. International Review of Hydrobiology, 2009, 94(6) : 656-672.

[26] Ye L, Cai QH. Forecasting daily chlorophyll a concentration during the spring phytoplankton bloom period in Xiangxi Bay of the Three-Gorges Reservoir by means of a recurrent artificial neural network. Journal of Freshwater Ecology, 2009,24 (4) : 609-617.

[27] Peng CQ, Chen L, Bi YH et al. Effects of flood regulation on phytoplankton community structure in the Xiangxi River, a tributary of the Three Gorges Reservoir. China Environmental Science, 2014, 34(7)：1863-1871. [彭成荣, 陈磊, 毕永 红等. 三峡水库洪水调度对香溪河藻类群落结构的影响. 中国环境科学, 2014, 34(7) : 1863-1871.]

[28] Yang M, Bi YH, Ai Y et al. A preliminary study on the effect of flow rate on the condition of artificial control in Xiangxi Bay phytoplankton water. Resources and Environment in the Yangtze Basin, 2012, 21(2): 220-224. [杨敏, 毕永红, 艾 鹰等. 人工控制条件下水流速对香溪河库湾浮游植物影响的初步研究. 长江流域资源与环境, 2012, 21 (2): 220-224.]

[29] Gao MY, Zhu KX, Bi YH et al. Spatiotemporal patterns of surface-suspended particulate matter in the Three Gorges Reservoir. Environmental Science and Pollution Research, 2016, 23(4) : 3569-3577.

[30] Temoka C, Wang JX, Bi YH et al. Concentrations and mass fluxes estimation of organochlorine pesticides in Three Gorges Reservoir with virtual organisms using in situ PRC-based sampling rate. Chemosphere, 2016, 144: 1521-1529.

[31] Deyerling D, Wang JX, Bi YH et al. Depth profile of persistent and emerging organic pollutants upstream of the Three Gorges Dam gathered in 2012/2013. Environmental Science and Pollution Research, 2016, 23(6) : 5782-5794. 
[32] Zhao YL, Zhang QQ, Liu XH et al. Influence of the Three Gorges Dam on the community structure and diversity of beauibu community in Xiangxi River. Journal of Hydroecology, 2017, 38(3) : 45-50. [赵媛莉, 张倩倩, 刘新华等. 三峡大 坝对香溪河底栖微生物群落结构和多样性的影响. 水生态学杂志, 2017, 38(3) : 45-50.]

[33] Zhang K, Xiong X, Hu HJ et al. Occurrence and characteristics of microplastic pollution in Xiangxi Bay of Three Gorges Reservoir, China.. Environmental Science \& Technology, 2017, 51(7) : 3794-3801.

[34] Mao N, Wang MH, Ho YS et al. A bibliometric study of the trend in articles related to risk assessment published in Science Citation Index. Human \& Ecological Risk Assessment an International Journal, 2010, 16(4) : 801-824.

[35] Lin JJ, Yang ZY, Liu D et al. Characteristics of grain size composition and nitrogen distribution in the sediment of the riparian zone of the Three Gorges under dry and wet cycles. Acta Pedologica Sinica, 2016, 53(3): 602-611. [林俊杰, 杨振 宇, 刘丹等. 干湿交替下三峡支流消落带沉积物粒径组成及氮分布特征. 土壤学报, 2016, 53(3) : 602-611.]

[36] Zhang B, Chen YP, Fang F et al. Nitrogen forms and their distribution characteristics in the soils of water-level-fluctuationg zone in the central Three Gorges Reservoir. Acta Scientiae Circumstantiae, 2012, 32(5) : 1126-1133. [张涁, 陈猷 鹏, 方芳等. 三峡库区淹没消落区土壤氮素形态及分布特征. 环境科学学报, 2012, 32(5): 1126-1133. ]

[37] Nie XQ, Guo Q, Li XL et al. Phosphorus forms and its spatiotemporal distribution characteristics in soil of water-level-fluctuating zone and upper margin of Xiangxi Bay. Environmental Science \& Technology, 2014, 37(11): 7-14. [聂小倩, 郭 强, 李晓玲等. 香溪河消落区及上缘土壤磷素的时空分布特征. 环境科学与技术, 2014, 37(11) : 7-14.]

[38] He LP, Fu C, Xie K et al. Characteristics of soil phosphorus storage in different types of fluctuation zones in Wanzhou section of the Three Gorges Reservoir Area. Resources and Environment in the Yangtze Basin, 2014, 23(4) : 534-541. [何立 平, 付川, 谢昆等. 三峡库区万州段不同类型消落带土壤磷形态它存特征. 长江流域资源与环境, 2014, 23(4): 534-541.]

[39] Gao JM, Zhang K, Chen YP et al. Occurrence of organotin compounds in river sediments under the dynamic water level conditions in the Three Gorges Reservoir Area, China. Environmental Science \& Pollution Research, 2015, 22(11): 8375-8385.

[40] Wang YC, Ao L, Lei B et al. Assessment of heavy metal contamination from sediment and soil in the riparian zone China's Three Gorges Reservoir. Polish Journal of Environmental Studies, 2015, 24( 5 ) : 2253-2259.

[41] Tao JL, Xu M, Huang SP et al. Time dynamic analysis of soil phosphorus in water-level-fluctuation zone of Xiangxi River in Three Gorges Reservoir area. Yangtze River, 2017, 48(15): 10-16. [陶佳丽, 徐萌, 黄生平等. 三峡库区香溪河消 落带土壤磷时间动态研究. 人民长江, 2017, 48(15) : 10-16.]

[42] Chen XJ, Pan XJ, Feng K et al. The research about the protozoa and rotifer community structure in Xiaojiang River backwater area. Journal of Hydroecology, 2012, 33(5): 31-35. [陈小娟, 潘晓洁, 冯坤等. 小江回水区原生动物和轮虫群 落结构特征研究. 水生态学杂志, 2012, 33(5) : 31-35.]

[43] Tian ZB, Liu DF, Yao XJ et al. The water temperature strtification of Xiangxi Bay group in the seasonal succession of phytoplankton functional effects. Resources and Environment in the Yangtze Basin, 2014, 23(5): 700-707. [田泽斌, 刘德 富, 姚绪姣等. 水温分层对香溪河库湾浮游植物功能群季节演替的影响. 长江流域资源与环境, 2014, 23(5): 700-707. ]

[44] Liu L, Liu DF, Xiao SB et al. Effects of thermal strtification on spring blooms in Xiangxi Bay of the Three gorges Reservoir. Environmental Science, 2012, 33(9) : 3046-3050. [刘流, 刘德富, 肖尚斌等. 水温分层对三峡水库香溪河库湾 春季水华的影响. 环境科学, 2012, 33(9):3046-3050.]

[45] Huang Y, Fan D, Liu D et al. Nutrient estimation by HJ-1 satellite imagery of Xiangxi Bay, Three Gorges Reservoir, China. Environmental Earth Sciences, 2016, 75(8): 1-13.

[46] Shang H, Jia L, Menenti M. Analyzing the inundation pattern of the Poyang Lake floodplain by passive microwave data. Journal of Hydrometeorology, 2015, 16(2): 652-667.

[47] Li J, Li DX, Wang XK. Three-dimensional unstructured-mesh eutrophication model and its application to the Xiangxi River, China. Journal of Environmental Sciences, 2012, 24(9): 1569-1578.

[48] Holbach A, Wang LJ, Chen H et al. Water mass interaction in the confluence zone of the Daning River and the Yangtze River-A driving force for algal growth in the Three Gorges Reservoir. Environmental Science \& Pollution Research, 2013, 20 (10): 7027-7037.

[49] Li YJ, Cheng Y, Wang YC, Parameter sensitivity analysis of water quality model for typical tributary in TGP reservoir area. Yangtze River, 2017, 48(16): 19-24. [李亚军, 程瑶, 王雨春. 三峡库区典型支流水质模型及其参数敏感性分 析. 人民长江, $2017,48(16): 19-24$.] 